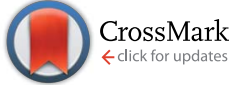

Cite this: RSC Adv., 2017, 7, 17806

Received 27th December 2016 Accepted 5th March 2017

DOI: $10.1039 / c 6 r a 28743 c$

rsc.li/rsc-advances

\title{
Highly efficient metal organic framework (MOF)- based copper catalysts for the base-free aerobic oxidation of various alcohols $\uparrow$
}

\begin{abstract}
Abu Taher, Dong Wook Kim and Ik-Mo Lee*
Copper (Cu) containing metal organic frameworks (MOFs) are found to be highly efficient heterogeneous catalysts for oxidation of various alcohols. A wide range of alcohols, including alcohols containing inactive hetero-aryl and long-chain alkyl units, are selectively converted into their corresponding products. The catalytic efficiency of the Cu containing MOFs, along with the co-catalyst 2,2,6,6-tetramethyl-piperidyl1-oxy (TEMPO), was demonstrated by the high conversion of the reactants with $100 \%$ selectivity under base-free conditions. The use of an inexpensive copper containing catalyst, the broad substrate scope, and the open air conditions and absence of base additive make this protocol very practical. The catalyst maintained a unique structural framework and it could be reused at least five times without significant loss of activity.
\end{abstract}

\section{Introduction}

In recent years, metal organic frameworks (MOFs) have been actively investigated and successfully used for different purposes in various branches of the pure and applied sciences. ${ }^{1}$ MOFs are three dimensional porous structures made of metal ions or clusters linked by organic molecules. The most attractive features of MOFs are their extremely high surface areas, as well as their tunable pore size and topology ${ }^{10}$ These key features, along with other characteristics, make them excellent candidates for a wide range of applications in heterogeneous catalysis. ${ }^{2}$ These catalysts can be readily recovered and reused, ${ }^{3}$ which is highly desirable for cost effectiveness and sustainability considerations in organic synthesis. In addition, copper $(\mathrm{Cu}),{ }^{4}$ as a classical transition metal, is considered to be one of the most attractive catalysts for use in the oxidation of alcohols due to its abundance of resources, low cost, non-toxic properties and high catalytic efficiency. In particular, $\mathrm{Cu}(\mathrm{II})$ complexes combined with TEMPO have been proven to have good catalytic performance for alcohol oxidation. ${ }^{5}$ Up to now, even though many efficient $\mathrm{Cu}(\mathrm{II}) / \mathrm{TEMPO}$ catalytic systems have been reported, ${ }^{6}$ there are only a limited number of studies on MOFbased $\mathrm{Cu}(\mathrm{II}) / \mathrm{TEMPO}$ systems for alcohol oxidation. ${ }^{7}$ However, $\mathrm{Cu}$ /TEMPO catalysts often require a large excess of base additives, which always results in a carboxylate product and basic

Department of Chemistry, Inha University, Incheon 402-751, South Korea. E-mail: imlee@inha.ac.kr; Fax: +82-32-867-5604; Tel: +82-32-860-7682

$\dagger$ Electronic supplementary information (ESI) available: Synthetic details, digital images, ${ }^{1} \mathrm{H}$ NMR spectra, EDS spectrum, $\mathrm{N}_{2}$ adsorption-desorption isotherms, pore volume vs. diameter curve, XPS spectra, X-ray powder diffraction, effect of catalyst loading and FTIR spectrum. See DOI: 10.1039/c6ra28743c waste disposal problems, ${ }^{8}$ as well as separation difficulties for catalytic recycling. ${ }^{9}$ Importantly, the presence of a base is not desirable from an industrial point of view due to corrosion, and it can adversely affect the stereoselectivity and cannot be used for a base-sensitive alcohol, ${ }^{10}$ a fact that is very important in the pharmaceutical industry. Interestingly, some Cu(II) complexes can catalyze alcohol oxidation by using TEMPO as a co-catalyst without base additives. ${ }^{11}$ Unfortunately, these copper catalysts are still homogeneous and are difficult to separate after the reaction.

On the other hand, the oxidation of alcohols to their corresponding carbonyl compounds is one of the most important reactions in organic synthesis, due to the wide-ranging utility of these carbonyl products as essential precursors and intermediates for the development of many drugs, fragrances, and fine chemicals. ${ }^{12}$ It traditionally requires numerous stoichiometric oxidants, and most of these alcohols are oxidized by toxic, corrosive and expensive oxidants, such as DMSO-coupled reagents, ${ }^{13}$ hypervalent iodines, ${ }^{14}$ and heavy-metal reagents. ${ }^{12 a, b}$ Unfortunately, these reactions result in large quantities of toxic materials and call for the use of stoichiometric quantities of moisture-sensitive, unrecoverable and expensive chemical reagents. Importantly, satisfactory results were obtained in only limited cases, in which a large excess of additives was required. Therefore, the development of potential catalysts for oxidation under mild conditions is highly desirable, but challenging, and a hot topic in both green chemistry and current organic synthesis. Herein, we report a highly efficient and reusable $\mathrm{Cu}(\mathrm{II} / \mathrm{MOF}$ (3) composite assisted by TEMPO, as the co-catalyst, for the selective oxidation of various alcohols to their corresponding products under mild conditions, without the assistance of any base additives (Scheme 1). 

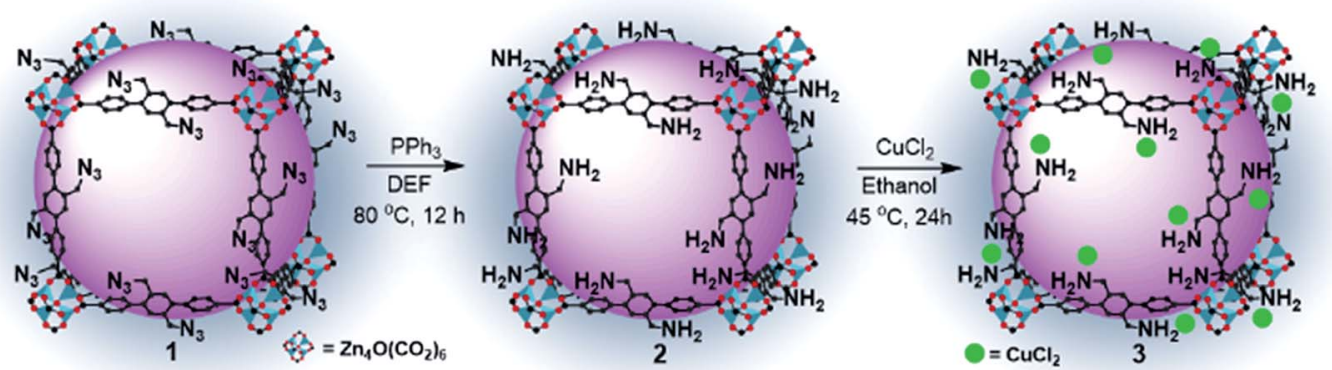

Scheme 1 Post-functionalization route of the $\mathrm{Cu}\left(\right.$ (I)/MOF- $\mathrm{NH}_{2}$ (3) composite.

\section{Results and discussion}

The azide-functionalized MOF- $\mathrm{N}_{3}$ (1) was prepared from the reaction of an organic linker, containing the azide functional group, with zinc nitrate hexahydrate in DEF, as described earlier. ${ }^{15}$ Subsequently, we tried to reduce the azide groups using triphenylphosphine $\left(\mathrm{PPh}_{3}\right)$ in DEF, as described in our earlier reports, and the detailed synthesis and characterization procedure of MOF- $\mathrm{NH}_{2}$ (2) is presented elsewhere. ${ }^{16}$ The FTIR and XRD spectra of the as-synthesized compounds 1 and 2 are similar to those of the defined compound, as shown in Fig. S2a and b, respectively (ESI $\dagger$ ). Moreover, an elemental analysis of $\mathbf{1}$ and 2 was in good agreement with their formula unit (Table S1, ESI $\dagger$ ). The catalyst 3 was synthesized by loading the $\mathrm{Cu}$ precursor, copper(II) chloride, into 2 in ethanol. The colour of the solution changed from green to colourless (Fig. 1f). After separating the MOF crystal by filtration, we dried the crystal under vacuum at $80^{\circ} \mathrm{C}$ to afford 3 as a green solid (Fig. S3, ESI $\dagger$ ). The copper complex was anchored on the amine-functionalized MOF layer through coordination, ${ }^{17}$ and the presence of the $\mathrm{Cu}$ complexes was examined by FTIR, HRSEM, TEM, XPS, EDS, TGA and ICP spectroscopic analyses. The loading of $\mathrm{Cu}$ in $\mathrm{MOF}-\mathrm{NH}_{2}$ was 5.02 wt\%, which was measured by inductively coupled plasma atomic emission spectroscopy (ICP-AES) and exactly matched the calculated value. The morphology of 3 was observed by SEM and TEM analyses. The HRSEM images at different magnifications are shown in Fig. 1a-c, and the TEM image of the catalyst 3 is shown in Fig. S4, (ESI $\dagger$ ). The SEM, and corresponding elemental mapping by energy-dispersive X-ray spectroscopic analysis (EDS) of 3, demonstrated the homogeneous distribution of copper and chloride ions in the aminefunctional MOF (Fig. 1d and e). The successful loading of $\mathrm{Cu}$ in 2 was confirmed by EDS spectroscopy. As shown in Fig. S5 (ESI $\dagger$ ), a clear $\mathrm{Cu}$ peak was resolved when we probed the selected area 1 on the $\mathrm{Cu}$ loaded MOF. Furthermore, the XPS survey spectra of 3 confirmed the existence of $\mathrm{Cu}$ and $\mathrm{Cl}_{2}$ in 3 (Fig. S6b $\dagger$ ). It can be remarked that the FTIR stretching band of the catalyst 3 at $1656 \mathrm{~cm}^{-1}$ is decreased, which may be attributed to the coordination interaction with $\mathrm{CuCl}_{2}$ (Fig. 2a). In particular, the amino group related peaks at $3373 \mathrm{~cm}^{-1}$ and $3290 \mathrm{~cm}^{-1}$ shifted to lower wave numbers at $3255 \mathrm{~cm}^{-1}$ and $3158 \mathrm{~cm}^{-1}$, respectively, in 3 because of the electron donation from the amino group to the $\mathrm{Cu}$ (II) center, which resulted in the formation of the Cu-amine functionalized-MOF complex. ${ }^{18}$ Furthermore, the characteristic XRD peak of $\mathrm{Cu}(112)$ at $2 \theta=$ $12.6^{\circ}$ is assigned due to the insertion of $\mathrm{Cu}^{2+}$ in 2 (Fig. 2b), which means that the structure of the amine-functionalized MOF has coordinated with $\mathrm{Cu}^{2+}$, and a new complex has been formed that is in accordance with earlier reports. ${ }^{19}$ In addition, it is found that after the yellow amine-functionalized MOF powders were added into the green copper(II) chloride containing ethanoic solution, the solution turned colourless (Fig. 1f), which further confirmed the likely formation of the MOF copper complex under the defined conditions. The XRD pattern of the reacted 3 crystals exhibited no apparent changes of the pattern from the starting 2 , as shown in Fig. $2 b$, and the
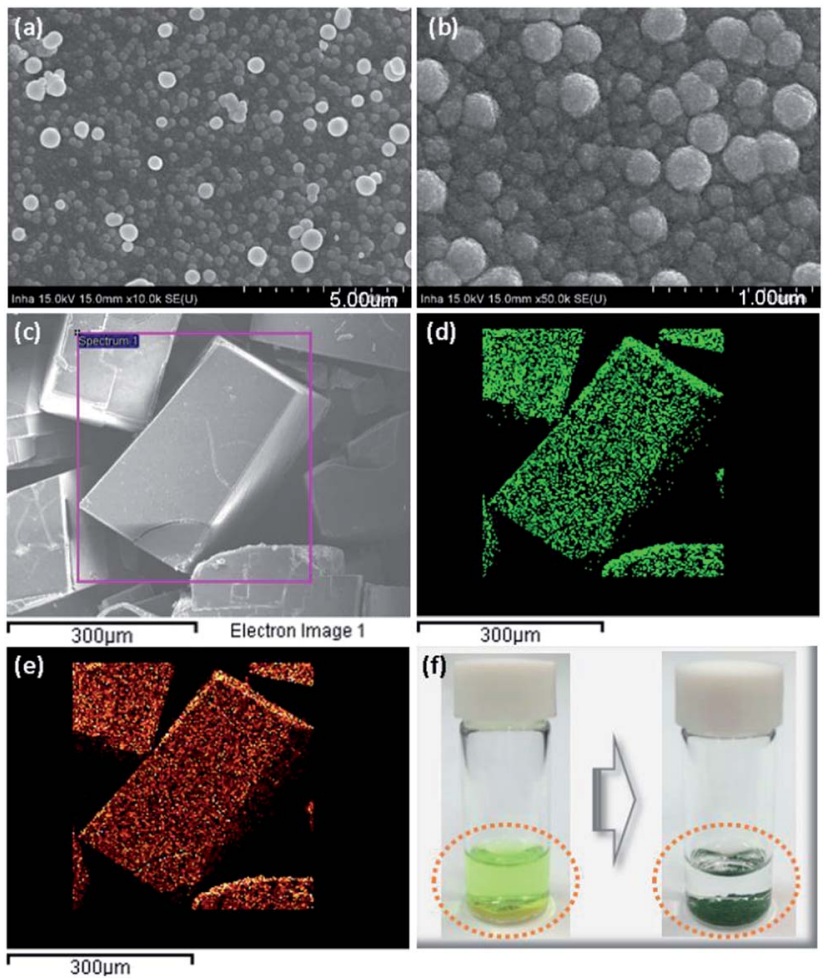

Fig. 1 SEM images of the surface of 3 at different magnifications (scale bars are shown at bottom left or right) for $5.00 \mu \mathrm{m}, 1.00 \mu \mathrm{m}$ and 300 $\mu \mathrm{m}$ respectively $(\mathrm{a}, \mathrm{b} \& \mathrm{c})$; the corresponding quantitative EDS mapping of $\mathrm{Cu}(\mathrm{d})$ and $\mathrm{Cl}(\mathrm{e})$; the color of the solution of catalyst 3 before and after reaction (f). 

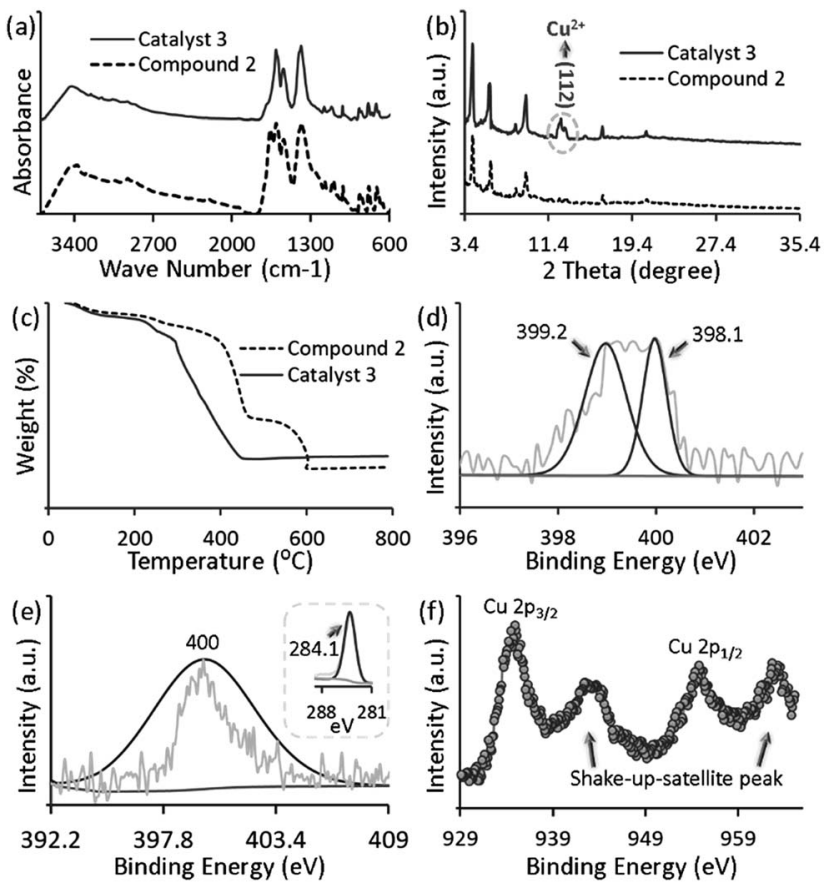

Fig. 2 (a) FTIR and (b) XRD spectra of compounds 2 and 3; TGA of compounds 2 and 3 (c); XPS spectra of the nitrogen $N$ 1s peaks for compound 2 (d) and for compound 3 (e); Cl S1 peaks for 3 (e, inset); XPS spectra of $\mathrm{Cu} 2 \mathrm{p}$ peaks of compound 3 (f).

C-O band was still maintained along with the $\mathrm{Zn}$ metal (Fig. S6a and b, ESI $\dagger$ ), which indicated that the modified MOF framework was constructed by zinc ions and carboxylate anions with a similar structural framework. Thermogravimetric analysis (TGA) indicates that $\mathbf{3}$ is thermally stable, and it shows thermal stability that is identical to the parent compound (Fig. 2c). Fig. S7 (ESI $\dagger$ ) shows the $\mathrm{N}_{2}$ adsorption-desorption isotherm profiles of 2 and 3. All of the adsorption-desorption isotherms show a type I shape, which is a characteristic of microporous materials. The BET surface area and pore volume of 2 were calculated to be $582.20 \mathrm{~m}^{2} \mathrm{~g}^{-1}$ and $0.31 \mathrm{~cm}^{3} \mathrm{~g}^{-1}$, respectively. Compared to 2 , the BET surface area and pore volume of 3 decreased to $156.04 \mathrm{~m}^{2} \mathrm{~g}^{-1}$ and $0.07 \mathrm{~cm}^{3} \mathrm{~g}^{-1}$, respectively, mainly because of the added mass of copper(II) chloride to 2 .

To gain further insights, we have also performed X-ray photoelectron spectroscopy (XPS) analysis. The $\mathrm{N}$ 1s spectrum is very useful for analyzing the nature of the $\mathrm{N}$ functionalities. Careful XPS analysis showed the presence of a split peak with maxima at 398.1 and $399.2 \mathrm{eV}$ for the functional groups of the primary amine $^{20}$ (Fig. 2d), whereas $\mathrm{N}$ 1s has a single peak at $400 \mathrm{eV}$ for the catalyst (Fig. 2e) which suggested strong coordination with copper(II) chloride. ${ }^{21}$ We also tried to determine the oxidation state of $\mathrm{Cu}$ in 3 using XPS. The $\mathrm{CuCl}_{2}$ related peak at $934.8 \mathrm{eV}$ was evident in the $\mathrm{Cu} 2 \mathrm{p}$ spectra of the catalyst, 3 (Fig. 2f). ${ }^{18}$ The spectra of $\mathrm{CuCl}_{2}$ in 3 was more clearly revealed in the $\mathrm{Cl}$ 1s spectra $(284.1 \mathrm{eV})$, as shown in Fig. 2 (e, inset). Furthermore, the $\mathrm{Cu} 2 \mathrm{p}$ XPS spectrum of $\mathrm{CuCl}_{2} / \mathrm{MOF}$ showed shakeup satellite peaks of the $\mathrm{Cu} 2 \mathrm{p}_{3 / 2}$ at 942 and $\mathrm{Cu} 2 \mathrm{p}_{1 / 2}$ at $962 \mathrm{eV}$, which confirmed the presence of $\mathrm{Cu}(\mathrm{II})$ species $\left(\mathrm{CuCl}_{2}\right)$
(Fig. 2f). ${ }^{22}$ Generally, the characteristic shakeup satellite is peculiar for the $\mathrm{Cu}$ (II) species, which relates to the $\mathrm{d}^{9}$ configuration of $\mathrm{Cu}$. Whereas, in the case of $\mathrm{Cu}(\mathrm{I})$, no satellite peaks can be observed because the screening via a charge transfer into the $\mathrm{d}$ states is not possible due to the presence of a completely filled d shell. ${ }^{22}$

The catalytic activity of the prepared catalyst 3 was tested with a representative set of primary and secondary benzylic and aliphatic alcohols. Initially, to investigate the catalytically active components of the $\mathrm{CuCl}_{2} / \mathrm{MOF}-\mathrm{NH}_{2}$ composite, a series of controlled experiments were carried out under exactly the same reaction conditions. The reactions were performed at $70{ }^{\circ} \mathrm{C}$, and under atmospheric pressure, using air as the oxidant under base-free conditions, and the results are summarized in Table 1. As shown in Table 1 (entry 1), 3 was an active catalyst for this reaction and $100 \%$ conversion (GC yield) of benzyl alcohol to benzaldehyde was achieved. On the contrary, 1 and 2 gave no conversion of benzyl alcohol (entries 2-4) in the absence of any catalyst, which confirmed the oxidation of benzyl alcohol to benzaldehyde is catalysed by $\mathrm{Cu}^{7}$ In addition, when only $\mathrm{CuCl}_{2}$ or a mixture of 2 and $\mathrm{CuCl}_{2}$ was used, relatively poor yields were obtained (entries 5 and 6). According to the literature, this may be due to the absence of coordinating ligands. ${ }^{23}$ Importantly, the results demonstrate that the connected ligand between the amine functionalized-MOF and the $\mathrm{Cu}$ moieties promoted the catalytic reaction of aerobic oxidation of benzyl alcohols under the defined conditions.

In order to obtain optimized catalytic performance, we investigated the effect of the solvent and found that $\mathrm{CH}_{3} \mathrm{CN}$ was the best solvent for this reaction (Fig. 3a). It is significant to note that 3 showed poor catalytic activity and the crystal structure deformed when water was used as the solvent (Fig. S9, ESI $\dagger$ ). Then, in a quick survey of the bases, the conversion of alcohol without using any base was similar to that when using $\mathrm{K}_{2} \mathrm{CO}_{3}$ as a base (Fig. 3b). In this catalytic system, the reason for the high efficiency without base is supposedly that 3 acts not only as a catalyst but also as a base, which has a much higher tendency to coordinate to copper to form the active species. So it might be thought that the MOF containing an amino group had adequate

Table 1 Aerobic oxidation of benzyl alcohol catalysed by various catalysts in $\mathrm{CH}_{3} \mathrm{CN}^{a}$

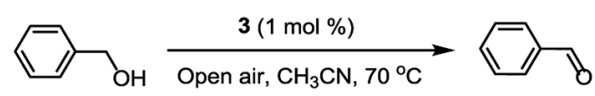

\begin{tabular}{llll}
\hline Entry & Catalyst & Time $(\mathrm{h})$ & Yield $^{b}(\%)$ \\
\hline 1 & $\mathrm{CuCl}_{2} @ M O F-\mathrm{NH}_{2}(3)$ & 6 & 100 \\
$2^{c}$ & MOF-N $_{3}(\mathbf{1})$ & 6 & 0 \\
$3^{c}$ & MOF-NH $_{2}(2)$ & 6 & 0 \\
4 & None & 6 & 0 \\
5 & ${\mathrm{MOF}-\mathrm{NH}_{2}+\mathrm{CuCl}_{2}}_{6}$ & 6 & 48 \\
6 & $\mathrm{CuCl}_{2}$ & 6 & 35
\end{tabular}

${ }^{a}$ Reaction conditions: benzyl alcohol $(1.0 \mathrm{mmol})$, TEMPO $(1.0 \mathrm{mmol})$, $\mathrm{CH}_{3} \mathrm{CN}(6 \mathrm{~mL})$ and air as an oxygen source. ${ }^{b} \mathrm{GC}$ yield. ${ }^{c}$ Calculated to have the same amount of $\mathrm{Zn}^{2+}$ (when comparing $\mathbf{3}$ with 1 and 2). 

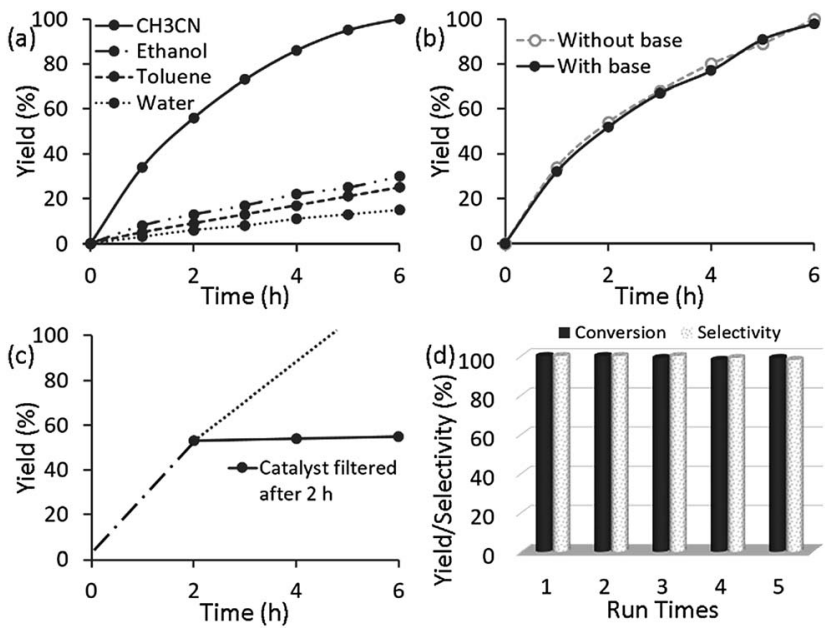

Fig. 3 Aerobic oxidation of benzyl alcohol catalysed by 3 (a) in different solvents, (b) with base and without base, and the (c) hot filtration test and (d) recyclability test. In all cases, the reaction conditions are the same as in Table 2 (entry 1 ).

basicity for deprotonation, and the oxidation could occur without any external base. ${ }^{24}$ Subsequently, we optimized the effect of the catalyst loading and found that $1 \mathrm{~mol} \%$ of $\mathrm{Cu}$ had the best efficiency for alcohol oxidation (Fig. S1†). These obtained results suggested that $3(1 \mathrm{~mol} \%)$ is required to promote base-free air oxidation in $\mathrm{CH}_{3} \mathrm{CN}$. With these optimized results on hand, the reactions of various aryl, alkyl and hetero-aryl substrates were investigated, with the assistance of TEMPO as the co-catalyst, and the results are summarized in Table 2.

In order to extend the scope of the aerobic oxidation by 3 , we performed the aerobic oxidation of various aromatic and aliphatic alcohols (Table 2). All of the reactions, such as for benzyl alcohol (entry 1), proceeded smoothly to give the corresponding products in high yield with 100\% selectivity under base-free conditions. Where possible, the product peaks were compared to those reported in the literature. ${ }^{25,26}$ The electronic nature of the substituents on the benzylic alcohol did have an effect on the reaction. Electron-donating group (EDG) containing substrates, such as $p$-tolylmethanol, (4-methoxyphenyl) methanol and (2-methoxyphenyl)methanol, reacted to give the products (entries 2-4) with yields (90-98\%) that are obviously higher than the yields for the electron-withdrawing group (EWG) containing substrates, such as (4-chlorophenyl)methanol, (2-chlorophenyl)methanol, (4-bromophenyl)methanol and (4-nitrophenyl)methanol (entries 5-8). Nevertheless, these results demonstrate that 3 successfully promoted the aerobic oxidation of various alcohols with either an EDG or EWG. It was noteworthy that the aerobic oxidation of secondary alcohols to their corresponding ketones is difficult, which is probably due to steric reasons. ${ }^{27}$ However, secondary alcohols such as 1phenylethan-1-ol, 1-( $p$-tolyl)ethan-1-ol and 1-(4-methoxyphenyl) ethan-1-ol also gave the expected products (entries 9-11) in good yield (93-98\%). The oxidation of heterocyclic alcohols, such as pyridin-3-yl-methanol and furan-2-yl-methanol, proceeded smoothly to provide the desired products (entries 12 and
Table 2 Aerobic oxidation of various alcohols catalysed by 3 in $\mathrm{CH}_{3} \mathrm{CN}^{a}$

Entry

${ }^{a}$ Reaction conditions: alcohol (1.0 mmol), TEMPO (1.0 mmol), $\mathrm{CH}_{3} \mathrm{CN}$ $(6 \mathrm{~mL})$ and air as an oxygen source. ${ }^{b}$ Isolated yield. Selectivity $100 \%$.

13) in moderate yield (58-61\%). In the case of the aliphatic alcohols, the product (entries 14-15) yields (48-65\%) were lower than those of the benzylic alcohols. In all cases, the catalyst exhibited good activity in the oxidation of various alcohols. Importantly, the results demonstrated that the selectivity of the primary alcohols along with the secondary alcohols was excellent $(100 \%$ selectivity), and the aliphatic alcohols showed similar trends.

In order to gain insight into the heterogeneous nature of $\mathbf{3}$, a leaching test was carried out, in which the solid catalyst was centrifuged and removed after $2 \mathrm{~h}$, and the reaction continued. As indicated in Fig. 3c, no further reaction took place without 3 
after initiation of the reaction at $4 \mathrm{~h}$, and the possible leaching of $\mathrm{Cu}$ after completion of the reaction was monitored by ICP analysis. This finding indicates that no leaching of the catalytically active sites occurs, and that 3 exhibits a typical heterogeneous catalyst nature, demonstrating that there was excellent coordination interaction between $\mathrm{Cu}^{2+}$ and the amine functionalized MOF. As a heterogeneous catalyst, 3 can be reused. After each catalytic cycle, 3 could be easily recovered by centrifugation or filtration, and directly reused in the next run under the same reaction conditions. It was observed that similar yields (98-100\%) were obtained by carrying out five runs without any significant loss of activity (Fig. 3d). More importantly, the catalyst maintained $100 \%$ selectivity during five consecutive runs. Evidently, the catalyst $\mathbf{3}$ is highly stable against the migration and sintering of $\mathrm{Cu}$ and exhibits excellent activity, selectivity, and stability for the oxidation of alcohols under mild reaction conditions without the assistance of any base additives. The FTIR, XRD and XPS spectra of the catalyst, 3, were similar to those of the fresh catalyst after recycling five times (Fig. S10 and S6c, ESI $\dagger$ ), which suggests that the structure and composition of 3 was maintained.

The well-defined pores of MOFs may offer unique advantages for catalytic applications, compared with other catalysts in which the pores may favor size and shape selective catalysis. ${ }^{3 b, 28}$ The pore size of the as-synthesized catalyst, $\mathbf{3}$, was bigger than all of the substrates, as shown in Table 2. For example, the size of benzyl alcohol is 6.9 by $4.9 \AA$, and the size of (4-methoxyphenyl)methanol is 9.0 by $4.9 \AA$ (Fig. 4), which is smaller than the pore size of 3 (18.3 $\AA$ ) as shown in Fig. S7 and S8† respectively. Therefore, this enhancement and the high selectivity would be due to the structure of the pores and surface functionality of the MOF frameworks.

\section{Experimental}

\section{Catalyst preparation}

MOF- $\mathrm{N}_{3}$ (1) was prepared according to the literature procedure, ${ }^{15}$ and the amine-functionalized MOFs (2) were synthesized by our earlier reported procedure. ${ }^{16}$ Typically, $0.31 \mathrm{~g}$ of MOF-N $\mathrm{N}_{3}$ crystals was placed in a $30 \mathrm{~mL}$ screw vial, and a solution of $0.80 \mathrm{~g}$ of triphenylphosphine $(3.0 \mathrm{mmol})$ in DEF $(30 \mathrm{~mL})$ was added. The vial was kept at $80^{\circ} \mathrm{C}$ in an oil bath for $10 \mathrm{~h}$ without stirring.

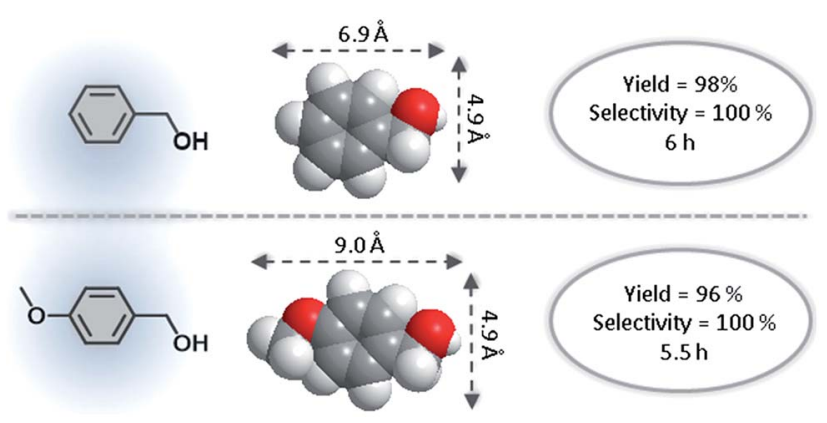

Fig. 4 Size and shape selectivity in the aerobic oxidation of benzyl alcohol and (4-methoxyphenyl)methanol using 3 .
After all the starting materials had disappeared (monitored by FTIR spectroscopy), MOF- $\mathrm{NH}_{2}$ was given as a slightly yellow solid (Fig. S3c, ESI $\dagger$ ). ${ }^{16}$ The crystals were filtered off and washed with fresh DEF $(20 \mathrm{~mL} \times 4)$ and ethanol, and then immersed in ethanol for $12 \mathrm{~h}$. The material was dried at $80{ }^{\circ} \mathrm{C}$ under vacuum for $24 \mathrm{~h}$. Finally, $0.04 \mathrm{~g}$ ( $0.30 \mathrm{mmol})$ of copper(II) chloride was dissolved in ethanol $(12 \mathrm{~mL})$, and $0.30 \mathrm{~g}$ of compound 2 was added to the solution in a $20 \mathrm{~mL}$ vial. The reaction mixture was kept in an oil bath at $45^{\circ} \mathrm{C}$ for $24 \mathrm{~h}$, and the color of the solution changed from blue to colorless (Fig. 1f, ESI $\dagger$ ). The crystals were filtered off and washed with fresh ethanol $(20 \mathrm{~mL} \times 4)$, and $\mathrm{Cu}(\mathrm{II}) / \mathrm{MOF}-\mathrm{NH}_{2}$ (3) was given as a blue solid (Fig. S3d, ESI $\dagger$ ). The material was dried at $80{ }^{\circ} \mathrm{C}$ under vacuum for $24 \mathrm{~h}$. Inductively coupled plasma (ICP) analysis showed $5.02 \%(\mathrm{wt})$ $\mathrm{Cu}$ in the resulting compound, $\mathbf{3}$.

\section{General procedure for the oxidation reaction}

The oxidation of alcohols was carried out using air oxygen (open air) at atmospheric pressure in a reaction vial. In a typical run, a solution of substrate $(1.0 \mathrm{mmol})$ and TEMPO $(1.0 \mathrm{mmol})$ in $\mathrm{CH}_{3} \mathrm{CN}$ ( $6 \mathrm{~mL}$ ) was added to $3(1 \mathrm{~mol} \%)$ without base, and the mixture was heated at $70{ }^{\circ} \mathrm{C}$ under atmospheric air. After completion of the reaction, the catalyst was filtered off and the conversion was measured using a GC (Agilent Technologies 7890A GC System). The crude product was obtained by concentrating the resultant solution under reduced pressure, and it was purified by column chromatography (on silica gel) using a mixture of EtOAc-hexane to give the pure corresponding product (Table 2). All the desired products are known compounds, which were characterized by comparison of their spectra with those reported in the previous literature. ${ }^{25,26}$ The separated catalyst was successively reused for the next reaction without any retreatment. A hot filtering experiment was carried out by separating the catalyst quickly from the reaction mixture after $2 \mathrm{~h}$ reaction time, and the filtrate was then maintained at $70{ }^{\circ} \mathrm{C}$ for an additional $4 \mathrm{~h}$.

\section{Conclusions}

In summary, we have developed a novel, highly efficient $\mathrm{Cu}$ (II) containing MOF catalytic system, which can be used with the co-catalyst TEMPO for alcohol oxidation. Numerous practical features associated with this method should facilitate its use in synthetic organic chemistry, including use without base additives, fast reaction rates, mild reaction conditions, and compatibility with $100 \%$ selectivity. More importantly, it is a highly active recyclable solid catalyst for the oxidation of various organic substrates under mild conditions. These results indicate that copper plays a central role in catalytic oxidation. Together, these catalyst systems provide compelling aerobic alternatives to traditional alcohol oxidation methods without the assistance of any base additives. Moreover, the exceptional reactivity of the novel catalyst system prompted our concurrent inquiry into the applications of these catalytic matrices in a multitude of catalytic reactions and engineering processes. 


\section{Acknowledgements}

This work is supported by the Inha University Research Grant (Inha 2016).

\section{Notes and references}

1 (a) A. U. Czaja, N. Trukhanb and U. Muller, Chem. Soc. Rev., 2009, 38, 1284-1293; (b) S. Keskin and S. Kızılel, Ind. Eng. Chem. Res., 2011, 50, 1799-1812; (c) H. Furukawa, K. E. Cordova, M. O’Keeffe and O. M. Yaghi, Science, 2013, 341, 1230444; (d) R. Ricco, C. Pfeiffer, K. Sumida, C. J. Sumby, P. Falcaro, S. Furukawa, N. R. Champness and C. J. Doonan, CrystEngComm, 2016, 18, 6532-6542.

2 (a) D. Farrusseng, S. Aguado and C. Pinel, Angew. Chem., Int. Ed., 2009, 48, 7502-7513; (b) A. Corma, H. Garcia and F. X. Llabrés i Xamena, Chem. Rev., 2010, 110, 4606-4655; (c) M. Yoon, R. Srirambalaji and K. Kim, Chem. Rev., 2012, 112, 1196-1231; (d) J. Gascon, A. Corma, F. Kapteijn and F. X. Llabrés i Xamena, ACS Catal., 2014, 4, 361-378; (e) J. W. Liu, L. F. Chen, H. Cui, J. Y. Zhang, L. Zhang and C. Y. Su, Chem. Soc. Rev., 2014, 43, 6011-6061.

3 (a) X. Feng, C. Xu, Z.-Q. Wang, S.-F. Tang, W.-J. Fu, B.-M. Ji and L.-Y. Wang, Inorg. Chem., 2015, 54, 2088-2090; (b) Y. Qi, Y. Luan, J. Yu, X. Peng and G. Wang, Chem.-Eur. J., 2015, 21, 1589-1597; (c) J. Wang, F.-W. Ding, J.-P. Ma, Q.-K. Liu, J.-Y. Cheng and Y.-B. Dong, Inorg. Chem., 2015, 54, 10865-10872.

4 (a) S. Pande, A. Saha, S. Jana, S. Sarkar, M. Basu, M. Pradhan, A. K. Sinha, S. Saha, A. Pal and T. Pal, Org. Lett., 2008, 10, 5179-5181; (b) S. E. Allen, R. R. Walvoord, R. P. Salinas and M. C. Kozlowski, Chem. Rev., 2013, 113, 6234-6237; (c) B. R. Kim, J. S. Oh, J. Kim and C. Y. Lee, Bull. Korean Chem. Soc., 2015, 36, 2799-2800; (d) Z. Miao, Y. Luan, C. Qib and D. Ramellac, Dalton Trans., 2016, 45, 1391713924; (e) B. R. Kim, J. S. Oh, J. Kim and C. Y. Lee, Catal. Lett., 2016, 146, 734-743.

5 (a) E. T. T. Kumpulainen and A. M. P. Koskinen, Chem.-Eur. J., 2009, 15, 10901-10911; (b) K. T. Mahmudov, M. N. Kopylovich, M. Silva, P. J. Figiel, Y. Y. Karabach and A. J. L. Pombeiro, J. Mol. Catal. A: Chem., 2010, 318, 44-50; (c) X. Zhang, W. Dong, Y. Luan, M. Yang, L. Tan, Y. Guo, H. Gao, Y. Tang, R. Dang, J. Li and G. Wang, J. Mater. Chem. A, 2015, 3, 4266-4273.

6 (a) P. Gamez, I. W. C. E. Arends, J. Reedijk and R. A. Sheldon, Chem. Commun., 2003, 2414-2415; (b) P. J. Figiel, A. Sibaouih, J. U. Ahmad, M. Nieger, M. T. Räisänen, M. Leskelä and T. Repo, Adv. Synth. Catal., 2009, 351, 2625-2632; (c) Q. F. Wang, Y. Zhang, G. X. Zheng, Z. Z. Tian and G. Y. Yang, Catal. Commun., 2011, 14, 92-95.

7 (a) A. Dhakshinamoorthy, M. Alvaro and H. Garcia, ACS Catal., 2011, 1, 48-53; (b) M. Paul, N. N. Adarsh and P. Dastidar, Cryst. Growth Des., 2014, 14, 1331-1337; (c) L. C. Li, R. Matsuda, I. Tanaka, H. Sato, P. Kanoo, H. J. Jeon, M. L. Foo, A. Wakamiya, Y. Murata and S. Kitagawa, J. Am. Chem. Soc., 2014, 136, 7543-7546.
8 (a) H. Miyamura, R. Matsubara, Y. Miyazaki and S. Kobayashi, Angew. Chem., Int. Ed., 2007, 46, 4151-4154; (b) H. Chen, Q. H. Tang, Y. T. Chen, Y. B. Yan, C. M. Zhou, Z. Guo, X. L. Jia and Y. H. Yang, Catal. Sci. Technol., 2013, 3, 328-338.

9 N. F. Zheng and G. D. Stucky, Chem. Commun., 2007, 38623864.

10 B. A. D’Sa, D. McLeod and J. G. Verkade, J. Org. Chem., 1997, 62, 5057-5061.

11 (a) M. F. Semmelhack, C. R. Schmid, D. A. Cortés and C. S. Chou, J. Am. Chem. Soc., 1984, 106, 3374-3376; (b) A. Cecchetto, F. Fontana, F. Minisci and F. Recupero, Tetrahedron Lett., 2001, 42, 6651-6653.

12 (a) R. A. Sheldon and J. K. Kochi, Metal-Catalyzed Oxidation of Organic Compounds, Academic Press, New York, 1981; (b) M. Hudlicky, Oxidations in Organic Chemistry, American Chemical Society, Washington, D.C., 1990; (c) R. A. Sheldon, I. W. C. E. Arends, G. J. Brink and A. Dijksman, Acc. Chem. Res., 2002, 35, 774-781; (d) J. Muzart, Tetrahedron, 2003, 59, 5789-5816; (e) M. Baumann and I. R. Baxendale, Beilstein J. Org. Chem., 2013, 9, 2265-2319.

13 B. A. Steinhoff, S. R. Fix and S. S. Stahl, J. Am. Chem. Soc., 2002, 124, 766-767.

14 (a) R. D. Ri-chardson and T. Wirth, Angew. Chem., Int. Ed., 2006, 45, 4402-4404; (b) M. Uyanik and K. Ishihara, Chem. Commun., 2009, 2086-2099; (c) M. Uyanik, M. Akakura and K. Ishihara, J. Am. Chem. Soc., 2009, 131, 251-262.

15 Y. Goto, H. Sato, S. Shinkai and K. Sada, J. Am. Chem. Soc., 2008, 130, 14354-14355.

16 A. Taher, D.-J. Lee, B.-K. Lee and I.-M. Lee, Synlett, 2016, 27, 1433-1437.

17 (a) R. K. Sharma, S. Dutta and S. Sharma, Dalton Trans., 2015, 44, 1303-1316; (b) A. Ghorbani-Choghamarani, Z. Darvishnejad and M. Norouzi, Appl. Organomet. Chem., 2015, 29, 170-175; (c) Q.-L. Yan, A. Cohen, N. Petrutik, A. Shlomovich, J.-G. Zhang and M. Gozin, ACS Appl. Mater. Interfaces, 2016, 8, 21674-21682.

18 (a) Y.-H. Choi and S.-H. Hong, Langmuir, 2015, 31, 81018110; $(b)$ K. J. Lee, Y.-I. Lee, I.-K. Shim, J. Joung and Y. S. Oh, J. Colloid Interface Sci., 2006, 304, 92-97.

19 Y. Zhang, Z. Xie, Z. Wang, X. Feng, Y. Wang and A. Wu, Dalton Trans., 2016, 45, 12653-12660.

20 A. Adenier, M. M. Chehimi, I. Gallardo, J. Pinson and N. Vila, Langmuir, 2004, 20, 8243-8253.

21 J. Marsh, L. Minel, M. G. Barthés-Labrousse and D. Gorse, Appl. Surf. Sci., 1998, 133, 270-286.

22 (a) M. Gopiraman, S. G. Babu, Z. Khatri, W. Kai, Y. A. Kim, M. Endo, R. Karvembu and I. S. Kim, Carbon, 2013, 62, 135-148; (b) M. Gopiraman, D. Deng, S. G. Babu, T. Hayashi, R. Karvembu and I. S. Kim, ACS Sustainable Chem. Eng., 2015, 3, 2478-2488.

23 (a) C. Bai, Q. Zhao, Y. Li, G. Zhang, F. Zhang and X. Fan, Catal. Lett., 2014, 144, 1617-1623; (b) A. Taher, D.-J. Lee and I.-M. Lee, Synlett, 2016, 27, 2333-2338.

24 S. Mannam, S. K. Alamsetti and G. Sekar, Adv. Synth. Catal., 2007, 349, 2253-2258. 
25 G. Zhang, X. Han, Y. Luan, Y. Wang, X. Wen and C. Ding, Chem. Commun., 2013, 49, 7908-7910.

26 C. Bai, A. Li, X. Yao, H. Liu and Y. Li, Green Chem., 2016, 18, 1061-1069.

27 (a) R. H. Liu, X. M. Liang, C. Y. Dong and X. Q. Hu, J. Am. Chem. Soc., 2004, 126, 4112-4113; (b) J. L. Long, X. Q. Xie,
J. Xu, Q. Gu, L. M. Chen and X. X. Wang, ACS Catal., 2012, 2, 622-631.

28 S. Horike, M. Dinca, K. Tamaki and J. R. Long, J. Am. Chem. Soc., 2008, 130, 5854-5855. 